\title{
The RISCK study: impact of the amount and type of dietary fat and carbohydrate on the plasma phospholipid-fatty acid status of free-living individuals
}

\author{
Julie Lovegrove ${ }^{1}$, Ana Rodriguez Mateos ${ }^{1}$, Gary Frost ${ }^{2}$, Bruce Griffin ${ }^{3}$, Tom Sanders ${ }^{4}$ \\ and Carmel Moore \\ ${ }^{1}$ University of Reading, Reading, UK, ${ }^{2}$ Imperial College, London, UK, ${ }^{3}$ University of Surrey, Guildford, UK, \\ ${ }^{4}$ Kings College, London, UK and ${ }^{5}$ MRC Human Nutrition Research, Cambridge, UK
}

The metabolic syndrome (MS) affects $\leq 25 \%$ of the UK population ${ }^{(1)}$ and confers substantial risk of CVD. A key scientific and public health question is whether reducing intakes of SFA via low-fat-high-carbohydrate (CHO) diets, or by moderate-fat diets in which SFA are substituted with MUFA, have differential effects on risk factors for MS. Moreover, there is a further question to be addressed in relation to the type of dietary $\mathrm{CHO}$, with current interest in the role of foods with a low glycaemic index (GI; LGI).

RISCK is a multi-centre randomised controlled dietary intervention study of 720 subjects at increased risk of MS. The study design has been previously reported ${ }^{(2)}$. After a run-in period of 4 weeks following the reference diet (high-SFA (HS)-high-GI (HGI) containing $38 \%$ energy ( $\% \mathrm{E}$ ) fat, $18 \% \mathrm{E}$ SFA, $10 \% \mathrm{E}$ MUFA, 6\% $\mathrm{E}$ PUFA) subjects were randomised to either the reference diet or one of four isoenergetic diets for 24 weeks (high-MUFA (HM)-HGI or HM-LGI) containing $38 \% \mathrm{E}$ fat, 10\%E SFA, 20\%E MUFA, 6\%E PUFA, or low-fat (LF)-HGI or LF-LGI containing $28 \% \mathrm{E}$ fat, $10 \% \mathrm{E}$ SFA, $11 \% \mathrm{E}$ MUFA, $6 \% \mathrm{E}$ PUFA). Total plasma phospholipid-fatty acid concentrations were determined at baseline and at the end of the study by GC analysis.

The total plasma phospholipid-fatty acid analysis was performed as a compliance measure to monitor the intake of dietary fat in the five intervention groups. Changes in the fatty acid profiles from the baseline visit to the follow-up visit were consistent with dietary prescription. The raised plasma phospholipid-MUFA levels (predominantly oleic acid) in the HM dietary groups appeared to increase at the expense of SFA. Changes in SFA and MUFA were driven by changes in palmitic and oleic acid respectively, which were the primary dietary sources of MUFA and SFA. No significant changes in other fatty acid classes such as $n-3$ PUFA, $n$ - 6 PUFA and trans-FA were observed.

Table. Plasma phospholipid-SFA and -MUFA levels (\% total fatty acids) at baseline and follow-up

\begin{tabular}{|c|c|c|c|c|c|c|c|c|c|c|}
\hline & \multicolumn{2}{|c|}{ HS-HGI } & \multicolumn{2}{|c|}{ HM-HGI } & \multicolumn{2}{|c|}{ HM-LGI } & \multicolumn{2}{|c|}{ LF-HGI } & \multicolumn{2}{|c|}{ LF-LGI } \\
\hline & Median & IQR & Median & IQR & Median & IQR & Median & IQR & Median & IQR \\
\hline \multicolumn{11}{|l|}{ SFA } \\
\hline$n$ & \multicolumn{2}{|c|}{78} & \multicolumn{2}{|c|}{106} & \multicolumn{2}{|c|}{103} & \multicolumn{2}{|c|}{102} & \multicolumn{2}{|c|}{109} \\
\hline Baseline & 44.3 & $43.4,45.4$ & 44.7 & $43.5,45.9$ & 45.0 & $43.7,45.7$ & 44.6 & $43.2,45.6$ & 44.6 & $43.2,45.6$ \\
\hline Follow-up & 44.3 & $43.3,45.5$ & 44.4 & $43.2,45.5$ & 44.4 & $43.3,45.5$ & 44.7 & $43.7,45.6$ & 44.7 & $43.5,45.8$ \\
\hline Change & 0.19 & $-0.15,0.53$ & -0.40 & $-0.72,-0.09$ & $\begin{array}{r}-0.42 \\
P=0 .\end{array}$ & $\begin{array}{l}-0.73,-0.12 \\
P=0.022 \dagger\end{array}$ & 0.18 & $-0.13,0.49$ & -0.19 & $-0.49,0.12$ \\
\hline \multicolumn{11}{|l|}{ MUFA } \\
\hline$n$ & \multicolumn{2}{|c|}{82} & \multicolumn{2}{|r|}{107} & \multicolumn{2}{|r|}{112} & \multicolumn{2}{|c|}{110} & \multicolumn{2}{|c|}{119} \\
\hline Baseline & 11.3 & $10.4,12.5$ & 11.6 & $10.4,12.5$ & 11.4 & $10.6,12.0$ & 11.5 & $10.7,12.5$ & 11.3 & $10.6,12.6$ \\
\hline Follow-up & 11.2 & $10.5,12.5$ & 12.4 & $11.0,13.6$ & 12.3 & $11.5,13.1$ & 11.8 & $10.7,12.6$ & 12.0 & $10.9,12.8$ \\
\hline \multirow[t]{2}{*}{ Change } & 0.07 & $-0.22,0.36$ & 0.82 & $0.56,1.09$ & 0.88 & $0.61,1.15$ & 0.18 & $-0.04,0.39$ & 0.27 & $0.04,0.49$ \\
\hline & \multicolumn{10}{|c|}{$P=0.0000^{*}, P=0.0000 \dagger$} \\
\hline
\end{tabular}

Mean change and 95\% CI (IQR) calculated on transformed scale, but expressed as change from median value at baseline; outliers first removed; Transformations: cubed (n-6 PUFA), $\log (n-3$ PUFA. *From ANCOVA of transformed baseline measures on transformed follow-up measures adjusted for gender, centre, ethnicity and baseline waist, $(\log )$ HDL, age; outliers first removed. $\dagger$ Also adjusted for weight change.

There was clear concordance between the plasma phospholipid-fatty acid levels and reported dietary intake data providing supporting evidence that the dietary fatty acid manipulation in the RISCK study was successfully achieved.

The RISCK study was funded by the Food Standards Agency (NO2031).

1. Tonkin $\mathrm{R}$ (2003) The $\mathrm{X}$ factor: obesity and the metabolic syndrome. http://www.the-ba.net/NR/rdonlyres/9DE73A6C-C61F-4007-93732BCBFC08F92B/0/Report.pdf

2. Jebb SA, Frost G, Griffin BA, Lovegrove JA, Moore C, Sanders T \& Williams CM (2007) Nutr Bull 32, 154-156. 